\title{
Performance Limitation Analysis of Highly-Digital Time-Based Closed-Loop Sensor-to-Digital Converter Architectures
}

\author{
Elisa Sacco, Student Member, IEEE, Jorge Marin, Johan Vergauwen, Member, IEEE, and \\ Georges Gielen, Fellow, IEEE
}

\begin{abstract}
This paper presents the theoretical and comparative analysis of two major time-based architectures for sensor interfaces. Both use a voltage-controlled oscillator (VCO) to achieve a highly-digital scalable implementation. The first architecture is based on a phase-locked loop, while the second one is countbased. Both systems are closed loop to efficiently mitigate the VCO nonlinearity. They show inherent first-order quantization noise shaping thanks to the use of an oscillator and phase detection. The two systems having a different working principle leads to different VCO requirements in terms of gain linearity (V-to-f or V-to-T linearity). Formulas are derived to predict the maximum SQNR for both architectures. Equations for the achievable maximum SNR taking into account the VCO phase noise are also derived, since this is the limit to the SNR in practical implementations. State-variable-based simulation results are presented, confirming the theoretical analysis and emphasizing the different design trade-offs and practical considerations.
\end{abstract}

\section{INTRODUCTION}

Distributed integrated sensors are increasingly important in the emerging era of the Internet of Things (IoT), as they are the gate between the physical world and the electronics. Lately, time-based sensor interfaces representing the signal by time information have become more and more popular: their highly-digital implementation allows a small area, a high energy efficiency and a good scalability exploiting all the benefits of advanced CMOS technology nodes [1].

Time-based implementations have been proposed both in open and closed loop. In [2] and [3], the oscillators are used as a frequency modulator, resulting in a $\Sigma \Delta$ modulator with no need for DACs due to the open-loop architecture. Two different analyses of such open-loop VCO-based ADC are presented in [4] and [5], emphasizing its similarity with pulse frequency modulators and $\Sigma \Delta$ modulators, respectively. However, in such open-loop systems the poor VCO linearity limits the overall system SNDR.

In [6]-[10] the VCO nonlinearity is tackled by placing the VCO in a closed loop after the passive/active analog integrator. Here, multiphase VCO outputs or VCOs in combination with counters are used as quantizers following power-hungry loop filters. PLL-based sensor-to-digital interface architectures have been described in [11]-[13]. In [14], a theoretical study shows

Manuscript received X XX, XXXX; revised X XX, XXX. The work was supported in part by VLAIO and Melexis.

E. Sacco, J. Marin and G. Gielen are with the MICAS Laboratories of the KU Leuven, Department of Electrical Engineering (ESAT), KU Leuven, 3001 Leuven, Belgium (e-mail: elisa.sacco@esat.kuleuven.be). J. Vergauwen is with Melexis, Tessenderlo, Belgium.

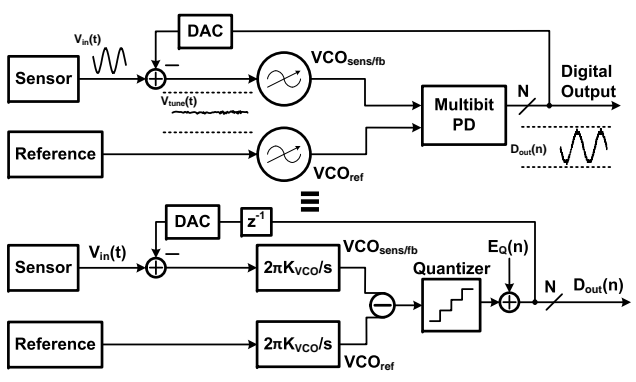

(a)

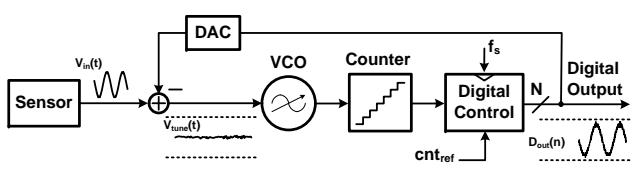

$\equiv$

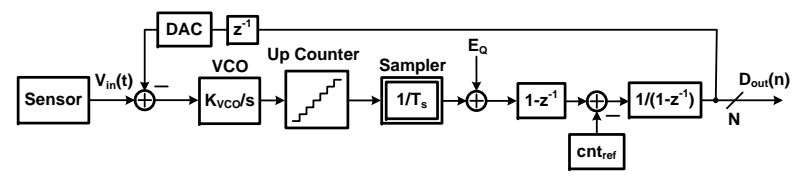

(b)

Fig. 1: Block diagram and corresponding simulation model of (a) the PLLbased and (b) the count-based closed-loop sensor interface architectures.

that the PLL-based sensor interface also resembles a first-order $\Sigma \Delta$ modulator due to the oscillator frequency-to-phase conversion. In these closed-loop systems the performance is rather limited by the VCO phase noise than the VCO nonlinearity.

This paper presents the theoretical analysis and simulation results of two major closed-loop highly-digital-oriented architectures used for sensor interfaces: the first is based on the phase-locked loop, as shown in Fig. 1(a), while the second is based on counting, as shown in Fig. 1(b). A closed loop is preferred for sensor interface applications due to the low-bandwidth and high-linearity requirements.

The paper is organized as follows. Section II highlights the working principles of the two architectures, leading to different VCO requirements. Section III derives the maximum SQNR and discusses the performance including phase noise. Simulation results employing a state-variable Matlab model confirm the theoretical analysis. Section IV draws conclusions. 


\section{SYSTEM-LEVEL SENSOR INTERFACE ARCHITECTURES}

\section{A. PLL-based Architecture}

Fig. 1(a) shows the basic building blocks of a PLL-based system used as a sensor interface [12]-[14]. The architecture consists of two matched VCOs and a multi-bit phase detector (PD). A time-domain chopping technique introduced in [13] is employed to cancel both the DC offset from the VCOs' mismatch and the VCOs' $1 / f$ noise. Note that the multi-bit PD can be substituted by a single-bit PD and a digital PI filter to create an $N$-bit output, resulting in an almost equivalent system [14], avoiding the multi-bit PD nonlinearity.

1) Working principle: The conversion is based on the locking of the two identical VCOs: one controlled by a reference voltage $\left(\mathrm{VCO}_{\text {ref }}\right)$ and one by a voltage determined by both the sensor and the feedback signal $\left(\mathrm{VCO}_{\text {sens/fb }}\right)$.

2) Locking condition: In every sampling period $T_{r e f}$, the oscillator $\mathrm{VCO}_{\text {sens/fb }}$ can lag or lead with respect to $\mathrm{VCO}_{\text {ref }}$. The PD outputs an $N$-bit code $\left(D_{\text {out }}\right)$, which is the result of time-domain quantization, and thus is the digitization of the sensor signal $\left(V_{i n}(t)\right)$. The locking condition implies that the $\mathrm{VCO}_{\text {sens/fb }}$ period is on average equal to the $\mathrm{VCO}_{\text {ref }}$ period:

$$
\bar{T}_{\text {sens } / f b}=T_{\text {ref }} \text {. }
$$

Hence, to ensure correct operation, the edge detection principle requires a voltage-to-period (V-to-T) linear VCO, modeled as follows:

$$
T_{V C O}=T_{0}-K_{T_{1}} \cdot V_{\text {tune }}
$$

where $T_{0}$ is the free-running period, $K_{T_{1}}$ is the linear V-to-T gain factor, and $V_{\text {tune }}$ is the VCO input voltage.

\section{B. Count-based Architecture}

Fig. 1(b) shows the basic building blocks of the count-based sensor-to-digital converter. The system consists of a VCO, a counter ${ }^{1}$, a sampling clock, digital blocks and a feedback circuit. Differently from [9], the integrator has been removed, leading to first-order noise shaping. Compared to [3], the loop has been closed, requiring further digital processing to ensure the correct signal and noise transfer functions.

1) Working principle: The basic operation is to count the number of VCO edges that occur within each sampling clock period $\left(T_{s}\right)$. The count values are then scaled according to a reference count value $\left(c n t_{r e f}\right)$, which can be recomputed to tackle PVT variations. Finally, a digital circuit computes the digital output $\left(D_{\text {out }}\right)$, which is fed back to the input.

2) Equilibrium condition: Due to the closed loop, the count values $\left(c n t_{\text {sens/fb }}\right)$ are on average equal to $c n t_{r e f}$, meaning that the oscillator frequency $\left(f_{\text {sens } / f b}\right)$ is kept on average proportional to the sampling frequency $\left(f_{s}\right)$ :

$$
\frac{\bar{f}_{\text {sens } / f b}}{f_{s}}=\overline{c n t}_{\text {sens } / f b}=c n t_{r e f} \text {. }
$$

\footnotetext{
${ }^{1}$ The counter may be replaced by a multi-phase VCO output allowing higher sampling frequencies. However, the phase noise performance of the two systems differs: the phase noise of the single-phase VCO employed in the count-based architecture is degraded by $10 \log _{2} N$ when used as $N$-phase VCO [5]. In this brief, in order to make a fair comparison between the two architectures, the same VCO is used: the count-based architecture is thus preferred. This leads to different sampling frequencies for the two systems.
}

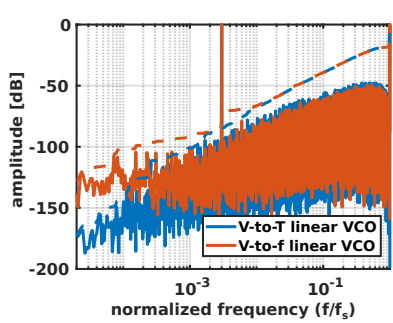

(a)

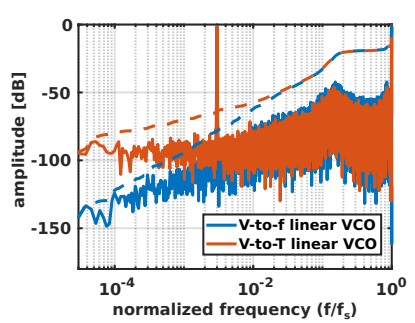

(b)
Fig. 2: Simulated output spectrum as a function of the input bandwidth with a V-to-f linear and a V-to-T linear VCO for (a) the PLL-based architecture $\left(T_{0}\right.$ $=100 \mathrm{~ns}, K_{T_{1}}=4 \mathrm{~ns} / \mathrm{V}, N=4, f_{s}=1 / T_{0}=10 \mathrm{MHz}$ ) and (b) the count-based architecture $\left(f_{0}=10 \mathrm{MHz}, K_{f_{1}}=8 \mathrm{MHz} / \mathrm{V}, N=4, f_{s}=1 \mathrm{MHz}\right)$. The dashed lines indicate the simulated integrated noise corresponding to the spectra with the same color.

\begin{tabular}{cc}
\hline V-to-T linear VCO & Equivalent V-to-f linear VCO \\
\hline$T_{0}$ & $f_{0}^{\prime}=1 / T_{0}=f_{0}$ \\
$K_{T_{1}}$ & $K^{\prime}{ }_{f_{1}}=-K_{T_{1}} / T_{0}{ }^{2}=-K_{T_{1}} \cdot f_{0}{ }^{2}$ \\
\hline V-to-f linear VCO & Equivalent V-to-T linear VCO \\
\hline$f_{0}$ & $T_{0}^{\prime}=1 / f_{0}=T_{0}$ \\
$K_{f_{1}}$ & $K^{\prime} T_{1}=-K_{f_{1}} / f_{0}{ }^{2}=-K_{f_{1}} \cdot T_{0}{ }^{2}$ \\
\hline
\end{tabular}

TABLE I: V-to-T and V-to-f linear VCO parameters.

Since the count value is directly proportional to the frequency, a voltage-to-frequency (V-to-f) linear VCO is required to ensure proper functionality. The VCO frequency is thus expressed as:

$$
f_{V C O}=f_{0}+K_{f_{1}} \cdot V_{\text {tune }}
$$

where $f_{0}$ is the free-running frequency, $K_{f_{1}}$ is the linear V-to-f gain factor, and $V_{\text {tune }}$ is the VCO input voltage. The ratio $f_{0} / f_{s}$ determines the reference count value $c n t_{r e f}(3)$.

\section{Analysis and comparison}

To verify the validity of these linearity conclusions and to analyze the performance of the two time-based architectures, a time-domain state-variable-based Matlab model has been developed for the two systems. At first, the phase noise is not included to focus on the impact of the VCO linearity relationship (V-to-T or V-to-f) on the maximum SQNR. The impact of phase noise will be discussed in Section III-B.

Fig. 2(a) and Fig. 2(b) plot the simulated output spectra of the PLL-based and the count-based architecture, respectively, employing a V-to-T (2) and a V-to-f (4) linear VCOs. The equations to compute the equivalent V-to-f linear VCO starting from a V-to-T linear $\mathrm{VCO}$, and vice versa, are shown in Table I. By employing a V-to-f linear VCO in the PLL-based architecture (Fig. 2(a)) and a V-to-T linear VCO in a count-based architecture (Fig. 2(b)), a noise floor appears in the spectrum limiting the ideal behavior. This confirms that in a PLL-based architecture a V-to-T linear VCO is needed to reach the maximum SQNR, while a count-based architecture requires a V-to-f linear VCO. To increase the legibility of the spectrum, the integrated noise is also plotted (dashed) [15].

In practical implementations, although the effect of the VCO nonlinearity is mitigated by the closed-loop architecture, 


\begin{tabular}{ccc}
\hline & PLL-based architecture & Count-based architecture \\
\hline$S_{i n, p-p}$ & $2 A K^{\prime}{ }_{f_{1}}$ & $\frac{2 \pi 2 A K_{f_{1}}}{f_{s}}$ \\
\hline$\Delta$ & $\frac{2 A K^{\prime}{ }_{f_{1}}}{2^{N}}$ & $2 \pi$ \\
\hline
\end{tabular}

TABLE II: Peak-to-peak signal power $S_{i n, p-p}$ and quantization step $\Delta$ definitions for both architectures where $A$ is the maximum signal amplitude, $N$ the quantizer resolution, $K^{\prime}{ }_{1}$ the equivalent VCO V-to-f gain (as the PLLbased architecture requires a V-to-T linear VCO), $K_{f_{1}}$ the VCO frequency gain for the count-based architecture, and $f_{s}$ the sampling frequency.

the use of a VCO with the non-optimal linearity relationship results in a performance $\operatorname{loss}^{2}$, as shown in Fig. 2.

\section{Performance Evaluation}

In the bottom parts of Fig. 1(a) and Fig. 1(b), the simulation block diagrams of both architectures are depicted. In the first subsection the ideal behavior of the two systems will be derived, showing the analogy with a first-order noise-shaped $\Sigma \Delta$ modulator operating in the phase/frequency domain instead of the voltage domain. In the second subsection the effect of the oscillator phase noise will be analyzed.

\section{A. SQNR and Oversampling}

The input signal power $P_{S}$ is equal to $\left(S_{i n, p-p} /(2 \sqrt{2})\right)^{2}$ where $S_{i n, p-p}$ is the peak-to-peak amplitude of the input signal. Assuming the quantization error has equal probability over the range of the quantization step $\Delta$, the quantization noise power can be written as $P_{Q}=\frac{\Delta^{2}}{12} \frac{\pi^{2}}{3}\left(\frac{1}{\mathrm{OSR}}\right)^{3}$. By definition, the SQNR is calculated as $P_{S} / P_{Q}$.

Table II reports the values of $S_{i n, p-p}$ and $\Delta$ for both architectures. By replacing these values in the aforementioned formulas for $P_{S}$ and $P_{Q}$, it is possible to derive the maximum SQNR equal to $6.02 N^{\prime}-3.41+30 \log (\mathrm{OSR})$. This is the well-known result for a $\Sigma \Delta$ modulator with first-order noise shaping. For the PLL-based architecture, $N^{\prime}$ corresponds to $N$, the number of bits in the PD, while for the count-based architecture, $N^{\prime}$ is defined as $N_{q}=\log _{2}\left(2 K_{f_{1}} A / f_{s}\right)$, and it also represents the quantizer resolution.

In the PLL-based architecture, the maximum SQNR depends only on $N$, the number of bits in the multi-bit quantizer. The system performance is not influenced by the VCO properties, except for the free-running frequency, which determines the system sampling rate. A change in the VCO gain, $K^{\prime}{ }_{f_{1}}$, does not have any impact on the ideal behavior. Moreover, the VCO gain and the number of bits in the feedback DAC can be chosen independently. On the other hand, in the count-based topology, the maximum SQNR strongly depends on the VCO gain, $K_{f_{1}}$, since the quantizer resolution, $N_{q}$, is function of $K_{f_{1}}$. Moreover, the number of bits in the feedback DAC, $N$, needs to be proportional to $N_{q}$ in order to ensure correct behavior. In both architectures, the quantizer resolution and the number of bits in the feedback DAC can be decoupled using a digital $\Sigma \Delta$ [9].

\footnotetext{
${ }^{2}$ The performance loss caused by the use of a VCO with the non-optimal linearity relationship depends on the number of bits in the quantizer. Increasing the number of bits allows to reduce the excited VCO frequency/period span, which appears more linear, hence decreasing the error.
}

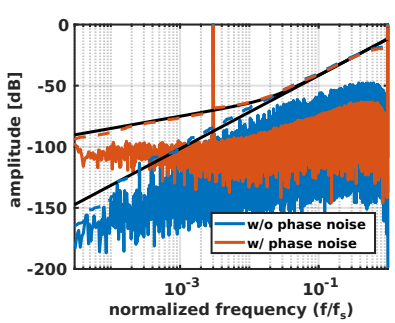

(a)

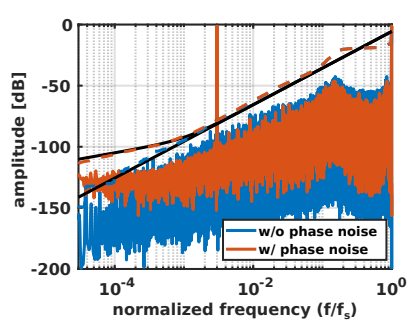

(b)
Fig. 3: Simulated power spectrum without and with phase noise $(-100$ $\mathrm{dBc} / \mathrm{Hz} @ 100 \mathrm{kHz}$ offset) for (a) the PLL-based architecture $\left(T_{0}=100 \mathrm{~ns}\right.$, $\left.K_{T_{1}}=40 \mathrm{~ns} / \mathrm{V}, N=4, f_{s}=1 / T_{0}=10 \mathrm{MHz}\right)$ and (b) the count-based architecture $\left(f_{0}=10 \mathrm{MHz}, K_{f_{1}}=8 \mathrm{MHz} / \mathrm{V}, N=4, f_{s}=1 \mathrm{MHz}\right)$. The dashed lines indicate the simulated integrated noise of the spectra with the same color. The black lines indicate the calculated theoretical results.

\section{B. SNR and Phase Noise}

The practically achievable SNR is limited by other sources of noise, such as the oscillator phase noise, beyond the quantization noise. The phase noise $L\left(\Delta f_{x}\right)$, expressed in $\mathrm{dBc} / \mathrm{Hz}$, represents the noise power density at an offset frequency $\Delta f_{x}$ from the carrier frequency $f_{0}$. The $1 / f^{2}$ phase noise power of the oscillator is:

$$
\begin{gathered}
P_{p n, f, P L L}=\pi L\left(\Delta f_{x}\right) \Delta f_{x}^{2} \cdot \frac{f_{0}}{\mathrm{OSR}} \\
P_{p n, \phi, C O U N T}=16 \pi L\left(\Delta f_{x}\right) \Delta f_{x}^{2} \cdot \frac{1}{f_{s}} \frac{1}{\mathrm{OSR}}
\end{gathered}
$$

for the PLL-based [14] and the count-based architectures [5], respectively. The free-running frequency of the VCOs, $f_{0}$, in the PLL-based architecture, and the clock frequency, $f_{s}$, in the count-based architecture represent the sampling rate of the two systems, respectively. The total noise power is then determined by the sum of the quantization noise power $P_{Q}$, derivable from Table II, and the phase noise power $P_{p n}$ (5) and (6). ${ }^{3}$

Fig. 3(a) and Fig. 3(b) plot the simulated power spectrum with and without phase noise for the PLL-based and the count-based architectures, respectively. Two regions can be distinguished: the first one where the phase noise dominates the quantization noise (flat spectrum), and the second one where the quantization noise dominates the phase noise (20dB/decade spectrum). In order to increase the legibility of the spectra and to compare the simulation results with the theoretical analysis, the integrated noises of the spectra are plotted in Fig. 3 as dashed line with the corresponding spectrum color. In both architectures, the simulated integrated noise is in agreement with the theoretical analysis, shown as black line, for both settings (with and without phase noise). In the simulations, the rms cycle-to-cycle jitter of the oscillator is calculated from the $1 / f^{2}$ phase noise using $\sigma_{c}^{2}=L\left(\Delta f_{x}\right) \Delta f_{x}^{2} / f_{0}^{3}[16]$.

Considering the PLL-based architecture, when the phase noise dominates, which is the typical operating region, $P_{p n}$

\footnotetext{
${ }^{3}$ Note that the formulas that express the signal power $P_{S}$ and the quantization noise power $P_{Q}$, both derivable from Table II, and the formulas for the phase noise power $P_{p n}(5)$ and (6) have the unit $\left[\mathrm{Hz}^{2}\right]$ for the PLL-based architecture, while being dimensionless for the count-based architecture.
} 


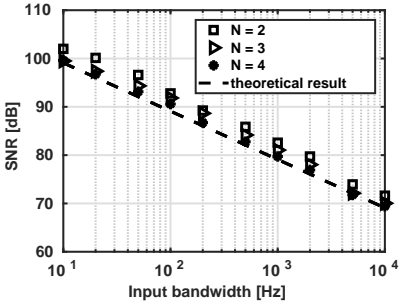

(a)

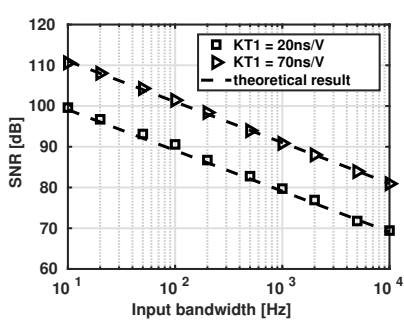

(b)

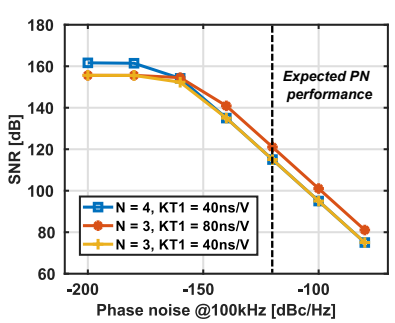

(a)

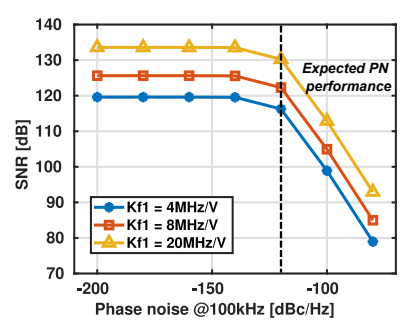

(b)

Fig. 4: SNR as a function of the input bandwidth for the PLL-based architecture with $-100 \mathrm{dBc} / \mathrm{Hz} @ 100 \mathrm{kHz}$ offset phase noise for (a) different numbers of bits in the multi-bit PD $\left(T_{0}=100 \mathrm{~ns}, K_{T_{1}}=20 \mathrm{~ns} / \mathrm{V}\right)$ and (b) different VCO period gains $\left(T_{0}=100 \mathrm{~ns}, N=4\right)$.

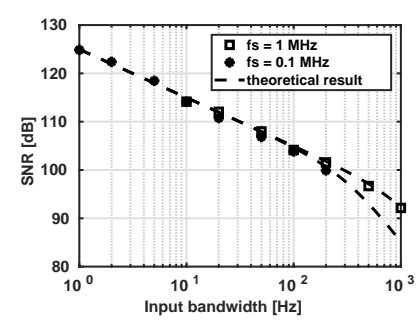

(a)

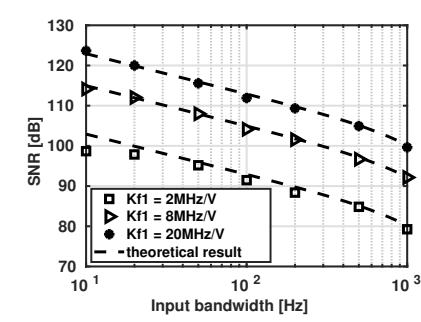

(b)

Fig. 5: SNR as a function of the input bandwidth for the count-based architecture with $-100 \mathrm{dBc} / \mathrm{Hz} @ 100 \mathrm{kHz}$ offset phase noise for (a) different sampling clock frequencies $\left(f_{0}=10 \mathrm{MHz}, K_{f_{1}}=8 \mathrm{MHz} / \mathrm{V}\right)$ and (b) for different VCO frequency gains $\left(f_{0}=10 \mathrm{MHz}\right)$.

is (much) larger than $P_{Q}$, the SNR can be equated as follows:

$$
\begin{aligned}
& \mathrm{SNR}_{p n, P L L}=20 \log \left(K^{\prime}{ }_{1} A / \sqrt{2}\right) \\
& -10 \log \left(\pi L\left(\Delta f_{x}\right) \Delta f_{x}^{2} f_{0}\right)+10 \log (\mathrm{OSR}) \\
& =10 \log \left(\frac{\left(K^{\prime}{ }_{f_{1}} A\right)^{2}}{\pi 4 L\left(\Delta f_{x}\right) \Delta f_{x}^{2} f_{i n}}\right),
\end{aligned}
$$

meaning that in the phase-noise-limited region of operation, increasing the number of bits $N$ does not increase the SNR by $6.02 \mathrm{~dB}$ for each bit. Fig. 4(a) indeed shows that simulation results for all number of bits $N$ are within few $\mathrm{dB}$ of the theoretical value (7). However, in contrast to the ideal case, the oscillator gain $K_{T_{1}}$ starts to influence the SNR performance when the phase noise is included, as shown in Fig. 4(b), confirming (7). The $+10 \log (\mathrm{OSR})$ behavior instead of $+30 \log (\mathrm{OSR})$ is also visible in the plots.

For the count-based topology, the VCO frequency gain $K_{f_{1}}$ plays an important role in determining the SNR in both the ideal and the non-ideal case. The phase-noise-limited SNR (when $P_{p n} \gg P_{Q}$ ) can be expressed as:

$$
\begin{array}{r}
\mathrm{SNR}_{p n, C O U N T}=20 \log \left(K_{f_{1}} A / \sqrt{2}\right) \\
-10 \log \left(4 / \pi L\left(\Delta f_{x}\right) \Delta f_{x}^{2} f_{s}\right)+10 \log (\mathrm{OSR}) \\
=10 \log \left(\frac{\pi\left(K_{f_{1}} A\right)^{2}}{16 L\left(\Delta f_{x}\right) \Delta f_{x}^{2} f_{i n}}\right) .
\end{array}
$$

As shown in Fig. 5(a), the SNR does not depend anymore on the sampling frequency $f_{s}$ for a given bandwidth. Increasing the VCO gain improves the SNR, as shown in Fig. 5(b).

Fig. 6: SNR as a function of the oscillator phase noise for (a) the PLL-based architecture $\left(T_{0}=100 \mathrm{~ns}\right)$ and (b) the count-based architecture $\left(f_{0}=10 \mathrm{MHz}\right.$, $f_{s}=1 \mathrm{MHz}, N=4$ ) for an input bandwidth of $100 \mathrm{~Hz}$ for different system parameter values (e.g. $N, K_{T_{1}}, K_{f_{1}}$ ).

However, the VCO gain strictly influences the achievable phase noise. A high-gain VCO usually shows a larger phase noise than a low-gain $\mathrm{VCO}$ at the same free-running frequency.

It is interesting to notice that in the PLL-based architecture operating in the phase-noise-limited region, the SNR (7) has lost its dependency on the number of bits $N$ (see Fig. 4(a)) compared to the maximum SQNR. Similarly, in the count-based architecture operating in the phase-noise-limited region, the SNR (8) has lost its dependency on the sampling frequency $f_{s}$ (see Fig. 5(a)) compared to the maximum SQNR. Moreover, both architectures show the same dependency on the VCO gain (see also Fig. 4(b) and Fig. 5(b)).

Fig. 6 summarizes the SNR results for both systems. In Fig. 6(a), for the PLL-based topology, it is visible how the number of bits, $N$, in the multi-bit PD influences the SNR when the phase noise is negligible (left part), while the VCO gain $K_{T_{1}}$ has no influence. On the other hand, when the phase noise starts dominating (right part), the VCO gain influences the SNR, while $N$ has no effect on the performance. Fig. 6(b) plots the SNR as a function of the phase noise for the count-based architecture, showing the effect of the oscillator gain $K_{f_{1}}$ in both regions (quantization noise dominant (left part) and phase noise dominant (right part)). The vertical dashed line limits the design space region, since practical $1 / f^{2}$ phase noise values for $\Delta f=100 \mathrm{kHz}$ and $f_{0}=10 \mathrm{MHz}$ are typically around or above $-120 \mathrm{dBc} / \mathrm{Hz}$.

In practical implementations, at very low bandwidths, which is the case for sensor interface applications, the system performance is limited by the oscillator phase noise ${ }^{4}$. It is, therefore, interesting to compare the performance of these two time-based architectures under this condition. Fig. 7 plots the SNR as a function of the input bandwidth for both the PLL-based and the count-based architectures. In order to have a fair comparison, the same VCO and phase noise are used in the simulations. As stated in Section II, the PLL-based architecture requires a V-to-T linear VCO, while the count-based architecture requires a V-to-f linear VCO. The proper VCO has been used in the simulations (Table I). The count-based architecture shows a $4 \mathrm{~dB}$ better SNR in the

\footnotetext{
${ }^{4}$ In this brief only the $1 / f^{2}$ oscillator noise is considered. The $1 / f$ noise can be canceled by means of chopping [13] in a PLL-based architecture. In a count-based architecture, the VCO flicker noise can be tackled by means of negative feedback [17].
} 


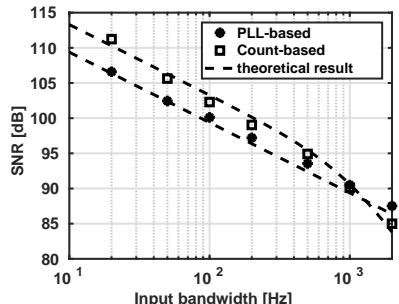

Fig. 7: SNR as a function of the input bandwidth for the PLL-based and the count-based architectures employing the same VCO $\left(f_{0}=10 \mathrm{MHz}, K_{f_{1}}=\right.$ $6.6 \mathrm{MHz} / \mathrm{V})$. The $\mathrm{VCO}$ phase noise is equal to $-100 \mathrm{dBc} / \mathrm{Hz} @ 100 \mathrm{kHz}$ offset.

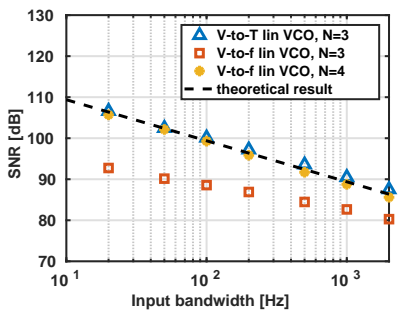

(a)

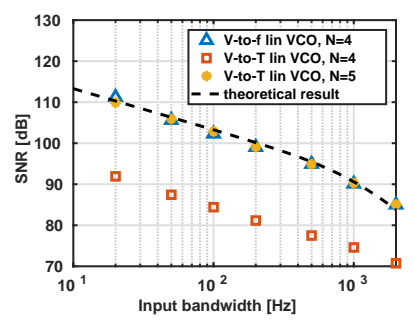

(b)
Fig. 8: SNR as a function of the input badwidth for (a) the PLL-based and (b) the count-based architectures employing the same VCO $\left(f_{0}=10 \mathrm{MHz}\right.$ $K_{f_{1}}=6.6 \mathrm{MHz} / \mathrm{V}$ ) but with a different linearity relationship (see Table I).

phase-noise-limited region (low bandwidth), confirming the theoretical result that is obtained by comparing (7) and (8) when the same VCO, the same phase noise and the same signal amplitude are used:

$$
\mathrm{SNR}_{p n, C O U N T}-\mathrm{SNR}_{p n, P L L}=10 \log \left(\pi^{2} / 4\right) \approx 4 \mathrm{~dB} .
$$

The transition from the phase-noise-limited to the quantization-noise-limited SNR is visible at high frequencies.

Fig. 8 shows that the use of the non-optimal VCO (see Sec. II) can further degrade the system performance (orange squares). However, by increasing the quantizer resolution, the excited range in the $\mathrm{VCO}$ characteristic reduces, hence appearing more linear, thus reducing the error due to using the non-optimal linearity relationship, which becomes negligible compared to phase noise. The optimization of $N$ depends on the system architecture, and the VCO gain and phase noise.

\section{Design guidelines}

The two time-based closed-loop architectures studied here theoretically have similar performance. However, the PLL-based system requires matched VCOs [14]; time-domain chopping relaxes this requirement [13]. The count-based topology intrinsically avoids this demand by employing only one VCO, which also leads to a lower power consumption. However, thanks to its pseudo-differential architecture, the PLL-based system is more robust against common-mode disturbances. This architecture is therefore suitable for differential sensors read-out, while the count-based system is a good candidate for interfacing single-ended sensors.

\section{CONCLUSION}

This paper has analyzed and compared the achievable performance of two major time-based closed-loop sensor interface architectures: PLL-based and count-based. The theoretical derivations have been validated by means of state-variable-based Matlab simulations. The different working principles of the two systems have been highlighted, leading to different VCO requirements in terms of gain linearity (V-to-T or V-to-f). Formulas describing the maximum SQNR have been derived for both architectures. Since the oscillator phase noise is the limitation in practice, equations to predict the practically achievable SNR have been derived and validated by simulations. Different trade-offs and considerations for the design of both architectures have been presented.

\section{ACKNOWLEDGMENT}

The authors would like to thank VLAIO for its financial support and Melexis for its technical and financial support.

\section{REFERENCES}

[1] G. Gielen et al., "Time-Based Sensor Interface Circuits in CMOS and Carbon Nanotube Technologies," IEEE Trans. Circuits Syst. I, vol. 63, no. 5, pp. 577-586, May 2016.

[2] M. Hovin, A. Olsen, T. S. Lande and C. Toumazou, "Delta-sigma modulators using frequency-modulated intermediate values," IEEE JSSC, vol. 32, no. 1, pp. 13-22, Jan 1997.

[3] J. Kim and S. Cho, "A time-based analog-to-digital converter using a multi-phase voltage-controlled oscillator," in Proc. ISCAS, pp. 3934-3937, May 2006.

[4] E. Gutierrez et al., "A Pulse Frequency Modulation VCO-ADC in 40nm," IEEE Trans. Circuits Syst. II, to be published.

[5] J. Kim, T. Jang, Y. Yoon and S. Cho, "Analysis and Design of VoltageControlled Oscillator Based Analog-to-Digital Converter," IEEE Trans. Circuits Syst. I, vol. 57, no. 1, pp. 18-30, Jan. 2010.

[6] M. Park and M. Perrott, "A 0.13m CMOS 78dB SNDR 87mW $20 \mathrm{MHz}$ BW CT ADC with VCO-based integrator and quantizer," in Proc. ISSCC, pp. 170-171,171a, 2009.

[7] S. Dey, K. Reddy, K. Mayaram and T. S. Fiez, "A 50 MHz BW 76.1 dB DR Two-Stage Continuous-Time DeltaSigma Modulator With VCO Quantizer Nonlinearity Cancellation," IEEE JSSC, vol. 53, no. 3, pp. 799813, March 2018.

[8] T. Kim, C. Han and N. Maghari, "A 4th-Order Continuous-Time DeltaSigma Modulator Using 6-bit Double Noise-Shaped Quantizer," IEEE JSSC, vol. 52, no. 12, pp. 3248-3261, Dec. 2017

[9] P. Prabha et al., "A Highly Digital VCO-Based ADC Architecture for Current Sensing Applications," IEEE JSSC, vol. 50, no. 8, pp. 1785-1795, Aug. 2015.

[10] K. Reddy, S. Dey, S. Rao, B. Young, P. Prabha and P. K. Hanumolu, "A $54 \mathrm{~mW} 1.2 \mathrm{GS} / \mathrm{s} 71.5 \mathrm{~dB}$ SNDR 50MHz BW VCO-based CT $\Delta \Sigma$ ADC using dual phase/frequency feedback in 65nm CMOS," in Proc. VLSI, pp. C256-C257, 2015.

[11] K. Lee, Y. Yoon and N. Sun, "A 1.8mW 2MHz-BW 66.5dB-SNDR ADC using VCO-based integrators with intrinsic CLA," in Proc. CICC, pp. 1-4, 2013.

[12] J. Van Rethy, H. Danneels, V. De Smedt, W. Dehaene and G. E. Gielen, "Supply-Noise-Resilient Design of a BBPLL-Based Force-Balanced Wheatstone Bridge Interface in 130-nm CMOS," IEEE JSSC, vol. 48, no. 11, pp.2618-2627, Nov., 2013.

[13] J. Marin, J. Van Rethy, J. Vergauwen and G. Gielen, "Digital-domain Chopping Technique for High-Resolution PLL-based Sensor Interfaces," Sensors and Actuators A: Physical, vol. 249, Aug., 2016.

[14] J. Van Rethy, H. Danneels and G. Gielen, "Performance analysis of energy-efficient BBPLL-based sensor-to-digital converters," IEEE Trans. Circuits Syst. I, Reg. Papers, vol. 60, no. 8, pp. 2130-2138, Aug. 2013.

[15] S. Pavan, R. Schreier, G. Temes, Understanding Delta-Sigma Data Converters, IEEE, 2017

[16] A. Demir, A. Mehrotra and J. Roychowdhury,"Phase noise in oscillators: a unifying theory and numerical methods for characterisation," IEEE DAC, pp. 26-31, 1998.

[17] P. Park, D. Ruffieux and K. A. A. Makinwa, "A Thermistor-Based Temperature Sensor for a Real-Time Clock With $\pm 2 \mathrm{ppm}$ Frequency Stability," IEEE JSSC, vol. 50, no. 7, pp. 1571-1580, July 2015. 